(1)

CrossMark

\title{
Role of anlotinib-induced CCL2 decrease in anti-angiogenesis and response prediction for nonsmall cell lung cancer therapy
}

\author{
Jun $\mathrm{Lu}^{1,2,8}$, Hua Zhong ${ }^{1,8}$, Tianqing Chu ${ }^{1}$, Xueyan Zhang ${ }^{1}$, Rong $\mathrm{Li}^{1}$, \\ Jiayuan Sun ${ }^{1}$, Runbo Zhong ${ }^{1}$, Yuqin Yang ${ }^{2}$, Mohammad Shah Alam ${ }^{3}$, \\ Yuqing Lou ${ }^{1}$, Jianlin $\mathrm{Xu}^{1}$, Yanwei Zhang ${ }^{1}$, Jun $\mathrm{Wu}^{4}$, Xiaowei $\mathrm{Li}^{3}$, \\ Xiaodong Zhao ${ }^{5,9}$, Kai Li ${ }^{6,9}$, Liming $\mathrm{Lu}^{2,7,9}$ and Baohui Han ${ }^{1,9}$
}

@ERSpublications

The underlying molecular mechanisms and predictive biomarkers of anlotinib benefitting NSCLC patients are still unclear. In this study, anlotinib-induced CCL2 decrease contributes to a novel insight for its anti-angiogenesis mechanism and responsive prediction. http://ow.ly/YZL930mXlfV

Cite this article as: $\mathrm{Lu} \mathrm{J}$, Zhong $\mathrm{H}$, Chu T, et al. Role of anlotinib-induced CCL2 decrease in antiangiogenesis and response prediction for nonsmall cell lung cancer therapy. Eur Respir J 2019; 53: 1801562 [https://doi.org/10.1183/13993003.01562-2018].

\section{ABSTRACT}

Background: Anlotinib has been demonstrated in clinical trials to be effective in prolonging the progression-free survival (PFS) and overall survival (OS) of refractory advanced nonsmall cell lung cancer (NSCLC) patients. However, the underlying molecular mechanisms and predictive biomarkers of anlotinib are still unclear.

Methods: A retrospective analysis of anlotinib administered to 294 NSCLC patients was performed to screen for underlying biomarkers of anlotinib-responsive patients. Transcriptome and functional assays were performed to understand the antitumour molecular mechanisms of anlotinib. Changes in serum CCL2 levels were analysed to examine the correlation of the anlotinib response between responders and nonresponders.

Results: Anlotinib therapy was beneficial for prolonging OS in NSCLC patients harbouring positive driver gene mutations, especially patients harbouring the epithelial growth factor receptor (EGFR) ${ }^{\mathrm{T} 790 \mathrm{M}}$ mutation. Moreover, anlotinib inhibited angiogenesis in an NCI-H1975-derived xenograft model via inhibiting CCL2. Finally, anlotinib-induced serum CCL2 level decreases were associated with the benefits of PFS and OS in refractory advanced NSCLC patients.

Conclusions: Our study reports a novel anti-angiogenesis mechanism of anlotinib via inhibiting CCL2 in an NCI-H1975-derived xenograft model and suggests that changes in serum CCL2 levels may be used to monitor and predict clinical outcomes in anlotinib-administered refractory advanced NSCLC patients using third-line therapy or beyond.

The ALTER-0303 trial is registered at ClinicalTrials.gov with identifier number NCT02388919.

This article has supplementary material available from erj.ersjournals.com

Received: April 102018 | Accepted after revision: Nov 292018

Copyright OERS 2019 


\section{Introduction}

Lung cancer is the leading cause of cancer-related death worldwide. The incidence of nonsmall cell lung cancer (NSCLC) is reported to be 39.05 per 10000 people, accounting for $\sim 85 \%$ of all lung cancer patients [1]. $\sim 75 \%$ of patients have advanced NSCLC that is unresectable at first diagnosis and has a 5-year survival rate of $<15 \%[2,3]$. Histologically, NSCLC consists mainly of squamous-cell carcinoma (LUSC) and adenocarcinoma (LUAD) [4]. First- and second-line therapies for advanced NSCLC include the inhibition of driver genes and systemic chemotherapy. For third-line therapy, there is no consolidated regimen [5]. Systemic chemotherapy for NSCLC includes conventional chemotherapeutic agents, inhibitors of angiogenesis and inhibitors of metastasis [6]. Although advanced NSCLC patients have obtained great benefits from first- and second-line therapies, drugs that potently prolong progression-free survival (PFS) and overall survival (OS) at third-line or over third-line therapy are still limited. Multitargeted drugs could introduce a new opportunity for prolonging PFS and OS in refractory advanced NSCLC patients who are receiving third-line or beyond third-line therapies [7-10].

Anlotinib is a multitargeted receptor tyrosine kinase inhibitor (TKI) with demonstrated antitumour effects in various cancers [8-12]. Previous studies have indicated that anlotinib inhibits tumour growth by inhibiting signalling pathways involving angiogenesis and cell proliferation $[8,13,14]$. Recombinant enzyme assays in vitro have indicated that anlotinib selectively inhibits vascular epidermal growth factor receptor (VEGFR)-1, -2 and -3 , platelet-derived growth factor receptor (PDGFR)- $\alpha$ and $-\beta$ and fibroblast growth factor receptor (FGFR)-1, $-2,-3$ and -4 , among others. Furthermore, anlotinib suppresses tumour cell growth via the inhibition of c-Kit, Ret, Aurora-B, c-FMS and DDR1 [15-17].

Recent clinical trials have indicated that anlotinib therapy prolongs PFS and OS in refractory advanced NSCLC subjected to third-line or beyond third-line therapy $[7,9,10]$. The underlying mechanism might be attributed to anlotinib-induced block of angiogenesis. However, the molecular mechanisms of the angiogenesis blocking are yet to be fully characterised. Furthermore, identifying a biomarker that can be used to monitor and predict the clinical response of advanced NSCLC patients to anlotinib is an urgent problem to be solved to improve clinical outcomes. Therefore, in the present study, we sought to explore the underlying anti-angiogenesis mechanism of anlotinib and identify possible biomarkers for monitoring and predicting the clinical outcomes of anlotinib therapy in advanced NSCLC patients receiving third-line or beyond third-line therapies.

\section{Methods}

\section{Patients and samples}

The clinical trials and sample preparations were performed according to our previous studies [7, 9]. The trial (ALTER-0303) is registered at ClinicalTrials.gov with identifier number NCT02388919.

\section{Anlotinib response stratification by subgroups}

According to the histopathological classification, all NSCLC patients were classified as LUSC or LUAD. Analysis of PFS and OS was performed based on the histopathological classification of all registered anlotinib-administered NSCLC patients. Classification of the positive and negative driver gene mutation (epithelial growth factor receptor (EGFR), ROS1 or ALK) was used to evaluate the response to anlotinib in LUAD patients. Furthermore, the subtypes of positive driver gene mutations were assessed to analyse correlations with PFS and OS. Finally, the number of metastases (stratified as more than three metastases and three or fewer metastases) in registered LUAD patients at the initial assessment was used to evaluate the response to anlotinib in LUAD patients.

\section{Cell experiments}

Cell culture procedures were performed according to our previous studies [18, 19]. For detailed methods on the cell viability analysis, flow cytometric analysis, cell invasion analysis, the wound-healing scratch assay and the cell immunofluorescence assay, refer to the supplementary materials.

Affiliations: ${ }^{1}$ Dept of Pulmonary Medicine, Shanghai Chest Hospital, Shanghai Jiao Tong University, Shanghai, China. ${ }^{2}$ Central laboratory, Shanghai Chest Hospital, Shanghai Jiao Tong University, Shanghai, China. ${ }^{3}$ Bio-ID Center, School of Biomedical Engineering, Shanghai Jiao Tong University, Shanghai, China. ${ }^{4}$ School of Life Science, East China Normal University, Shanghai, China. ${ }^{5}$ Shanghai Center for Systems Biomedicine, Shanghai Jiao Tong University, Shanghai, China. ${ }^{6}$ Dept of Thoracic Oncology, National Clinical Research Center for Cancer, Tianjin Medical University Cancer Institute and Hospital, Tianjin, China. ${ }^{7}$ Shanghai Institute of Immunology, Shanghai Jiao Ton University School of Medicine, Shanghai, China. ${ }^{8}$ These authors contributed equally: Jun Lu and Hua Zhong. ${ }^{9}$ These authors contributed equally: Xiaodong Zhao, Kai Li, Liming Lu and Baohui Han.

Correspondence: Baohui Han, Department of Pulmonary Medicine, Shanghai Chest Hospital, Shanghai Jiao Tong University, Shanghai 200030, China. E-mail: xkyyhandgmail.com 
RNA-seq

RNA-seq and bioinformatics analysis assays were performed according to our previous studies [18, 19]. Detailed information is provided in the supplementary materials. The raw data for this study are available in the EMBL database under accession number E-MTAB-5997: www.ebi.ac.uk/arrayexpress/.

\section{Animal experiments}

Experiments using Balb/c nude mice (Shanghai Laboratory Animals Center, Shanghai, China) were performed according to national guidelines for welfare. Procedures for the NCI-H1975-derived xenograft model induction, grouping, drug administration, tumour volume measurements and sample collection are described in the supplementary materials.

\section{Quantitative analysis}

Histology analysis (including hematoxylin and eosin (H\&E) staining and immunofluorescent histochemistry analysis), mRNA expression analysis and ELISA analysis were performed as conventional procedures. Detailed information is provided in the supplementary materials.

\section{Anlotinib responder analysis prediction}

We aimed to understand whether the changes in serum CCL2 levels could be used to predict the clinical response of NSCLC patients treated with anlotinib. 437 NSCLC patients enrolled in ALTER-0303 study, and 294 patients received anlotinib therapy. Of the 294 patients, there were 14 anlotinib responders (PFS $>80$ days) with qualified blood samples at baseline, best response and progressive disease, simultaneously. For comparing the differences between responders and nonresponders, we picked out randomly 14 anlotinib nonresponders (PFS $\leqslant 80$ days) with qualified blood samples at the baseline and progressive disease time points. The driver gene mutation status of NSCLC patients at their first biopsy is shown in supplementary table S1. Serum was isolated from blood according to standard methods. CCL2 levels were detected via ELISA as previously described. CCL2 levels in NSCLC patients were compared at different time points. Changes in CCL2 levels after anlotinib treatment were calculated based on CCL2 levels at different time points. Anlotinib responder analysis prediction was performed based on changes in CCL2 levels.

\section{Statistical analyses}

PFS and OS were summarised as median values and two-sided 95\% confidence intervals and were analysed using the Kaplan-Meier method. The Mantel-Cox test was used to perform the Kaplan-Meier survival analysis in GraphPad Prism 5 (San Diego, CA, USA). All in vitro experiments, excluding RNA-seq, were performed on at least three biological replicates. Data are presented as mean \pm SD. The data were examined using the two-tailed t-test, log-rank test, or one-way ANOVA with post hoc Bonferroni correction. Differences were considered significant at $\mathrm{p}<0.05, \mathrm{p}<0.01$ and $\mathrm{p}<0.001$.

\section{Results}

LUAD patients harbouring positive driver gene mutations have a better OS after anlotinib therapy

All NSCLC patients $(n=294)$ received anlotinib therapy for two consecutive weeks and then discontinued for one week (figure 1a) until progression of disease (PD) was observed. To examine the anlotinib response of NSCLC patients, we divided the patients into LUSC $(n=54)$ and LUAD $(n=240)$ according to the histopathologic classification. Kaplan-Meier analysis suggested that there was no difference in the PFS and OS curves between LUSC and LUAD after anlotinib therapy (supplementary figure S1a). Among the registered patients, LUAD accounted for the majority of all NSCLC patients. Therefore, we performed a subgroup analysis of the LUAD patients. Compared with the OS of the driver gene-negative patients $(n=147)$, that of the driver gene-positive patients $(n=93)$ was significantly longer after anlotinib therapy. However, there was no significant difference when the PFS analysis was performed (figure $1 \mathrm{~b}$ and $\mathrm{c}$ ). Subsequently, Kaplan-Meier analysis was performed to examine the correlations between mutation types of the driver gene and PFS or OS. The patients harbouring the ROS1 or EGFR ${ }^{\mathrm{T} 790 \mathrm{M}}$ mutation had a better response to anlotinib than those with other types of driver gene mutation (figure 1d and e). Further analysis suggested that the OS of patients harbouring the EGFR ${ }^{\mathrm{T} 790 \mathrm{M}}$ mutation was significantly longer than those with other driver gene types, although differences in PFS remained nonsignificant (figure $1 \mathrm{f}$ and g). Furthermore, the NSCLC patients harbouring more than three metastases had a shorter PFS and OS than the NSCLC patients harbouring three or fewer metastases (supplementary figure S1b). Interestingly, we found that some LUAD patients without a driver gene mutation also had a good response to anlotinib therapy (supplementary figure S1c-e), suggesting potential alternative biomarkers for distinguishing anlotinib responders from nonresponders. 

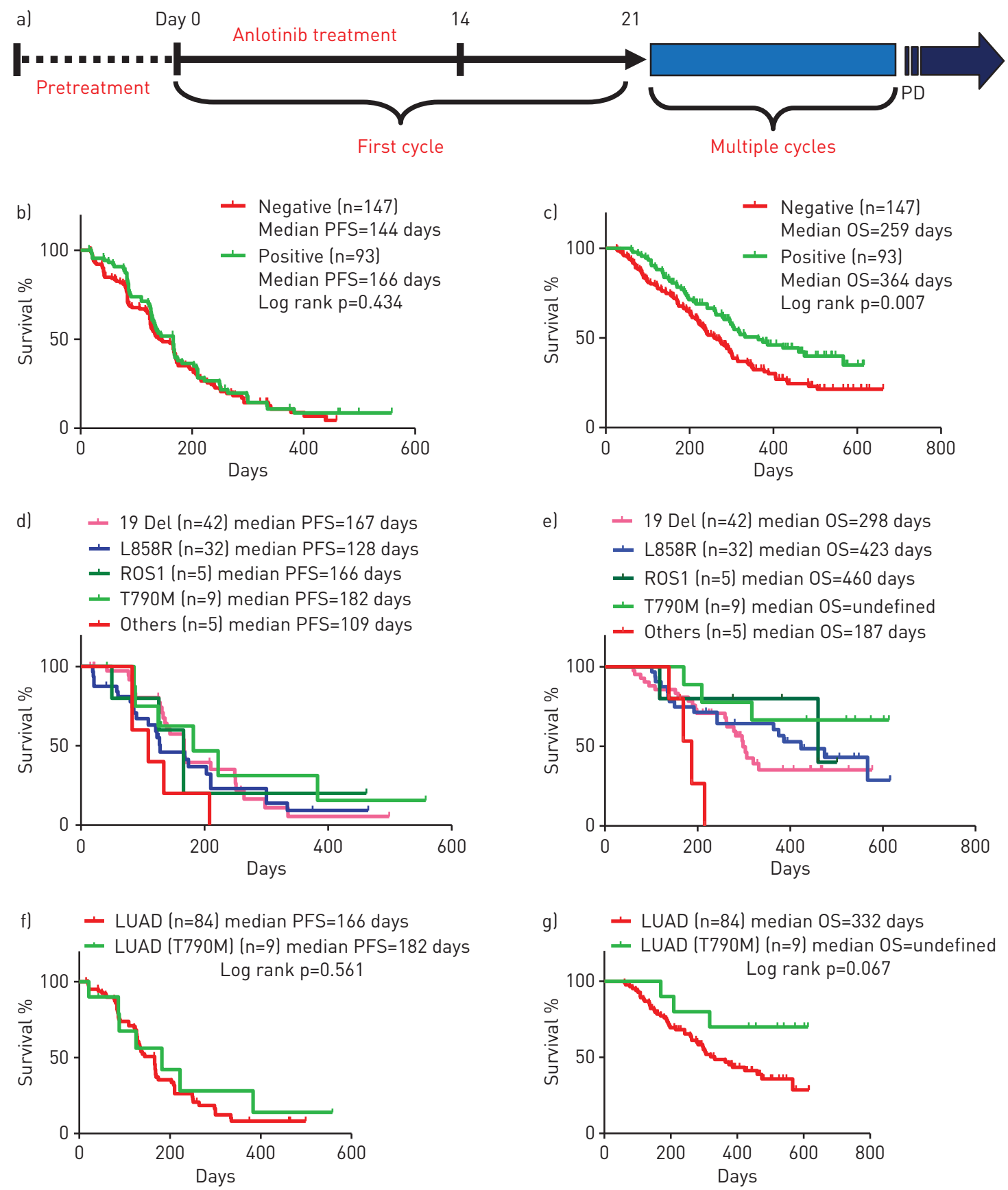

FIGURE 1 A retrospective analysis of anlotinib phase III clinical trial indicates that the overall survival of lung adenocarcinoma (LUAD) patients is associated with the driver gene mutation type. a) Timeline of the anlotinib phase III clinical trial. Kaplan-Meier plots of b) progression-free survival (PFS) and c) overall survival (OS) in nonsmall cell lung cancer (NSCLC) patients receiving anlotinib therapy by negative ( $\mathrm{n}=147$ ) and positive ( $\mathrm{n}=93$ ) driver gene mutations. Median (95\% CI) PFS 147 (131-163) days versus 166 (143-189) days, median (95\% CI) 0S 259 (234-284) days versus 364 (332-396) days. Analysis of d) PFS and e) OS in anlotinib-treated LUAD patients harbouring epidermal growth factor receptor (EGFR) and other positive mutations. Kaplan-Meier plots of f) PFS and g) OS in driver gene-positive LUAD patients receiving anlotinib therapy by $\mathrm{EGFR}^{\mathrm{T} 790 \mathrm{M}}$-negative $(\mathrm{n}=84)$ and $\mathrm{EGFR}^{\mathrm{T} 90 \mathrm{M}}$-positive $(\mathrm{n}=9)$ mutations. Median (95\% CI) PFS 166 (144-188) days versus $182(53-311)$ days, median 0S (95\% CI) 332 (299-365) days versus undefined. PD: progression of disease.

\section{Anlotinib induces $\mathrm{NCl}-\mathrm{H} 1975$ cell cytotoxicity in vitro and in vivo}

Understanding the underlying molecular mechanisms could be a prerequisite to identify valuable biomarkers for anlotinib responders. Here, we found that NCI-H1975 cells were the obviously sensitive to anlotinib in vitro, as demonstrated by cell apoptosis induction, cell invasion inhibition, cell cycle arrest and cell migration inhibition (supplementary figure S2a-i). These results suggested multiple cytotoxic effects of anlotinib upon NCI-H1975 cells. To examine whether anlotinib could inhibit tumour growth in vivo, we administered anlotinib in NCI-H1975-derived xenograft models. The timeline of the in vivo 
experiment is shown in figure 2a. After one cycle of anlotinib treatment, tumour growth was significantly inhibited, and the survival time was markedly enhanced, with acceptable toxicity at both dosages (figure $2 b$ and $c$ and supplementary figure S2j-1). Extensive tissue necrosis, evaluated by H\&E staining, was found in anlotinib-treated NCI-H1975-derived xenograft models, and the tumour cavity, as evaluated using computed tomography, revealed refractory advanced NSCLC patients after anlotinib therapy (figure $2 \mathrm{~d}$ and supplementary figure S1c and d), indicating that anti-angiogenesis could play an important role in anlotinib-induced tumour inhibition.

\section{Transcriptome analysis indicates that anlotinib-induced CCL2 blockade is involved in} anlotinib-induced angiogenesis block

Based on the observations of cytotoxicity and tissue necrosis induced by anlotinib, we next sought to understand the underlying molecular mechanisms via transcriptome-wide differential gene expression analysis (figure 2e). The results suggested that multiple biological processes or signalling pathways are involved in anlotinib-induced cytotoxicity (figure $2 \mathrm{f}$ and supplementary figure S3). Interestingly, among the downregulated genes, we found that certain angiogenesis-related biological processes (including angiogenesis, positive regulation of endothelial cell differentiation and microtubule processes) were the most enriched (figure $2 \mathrm{f}$ ).

CCL2 is an important angiogenesis target for tumour therapy [20]. Here, we found that CCL2 transcription was downregulated more than 17 -fold after anlotinib treatment in vitro (figure $2 \mathrm{~g}$ and $\mathrm{h}$ ). Analysis of the TCGA cohort suggested that high CCL2 expression significantly correlated with reduced survival in NSCLC patients (supplementary figure S4a and b). However, the gene coexpressed with CCL2 in LUAD, IL6, was not associated with anlotinib-induced anti-angiogenesis (supplementary figure S4c-g). Therefore, we performed a series of assays to characterise CCL2 expression in vitro and in vivo. CCL2 levels in the medium were significantly decreased after exposure of NSCLC cell lines (NCI-H1975, NCI-H2228, A549, NCI-H1650 and NCI-H2228) to anlotinib (figure 2i and supplementary figure S5). CCL2 levels both in serum and tumour tissue also markedly decreased after anlotinib administration (figure $2 \mathrm{j}$ and $\mathrm{k}$ ). Based on these observations, we hypothesised that anlotinib-induced anti-angiogenesis might be mainly attributed to the reduced CCL2 content.

\section{Anlotinib inhibits MMP9 expression in vitro and in vivo}

Previous studies have reported that the downregulation of CCL2 inhibits protein expression of angiogenesis-related signalling pathway in vitro and in vivo [20, 21]. In the present study, we found that among the matrix metalloproteins (MMPs) (including MMP1, MMP2, MMP3, MMP9 and MMP19), the mRNA expression of MMP9 was nearly blocked after anlotinib treatment in vitro and in vivo (figure 3a). Moreover, MMP9 protein was markedly decreased after anlotinib treatment (figure $3 b$ and c). Thus, proliferation and vascularisation were significantly inhibited after anlotinib administration (figure $3 \mathrm{~d}-\mathrm{g}$ ). Because high MMP9 levels contribute to tumour vascularisation [22], our results suggest that anlotinib-induced anti-angiogenesis possibly attributed to the inhibition of the CCL2-MMP9 axis.

\section{Anlotinib inhibits angiogenesis via the CCL2-MMP9 axis}

To further understand the role of CCL2 in anlotinib-induced anti-angiogenesis, human recombinant CCL2 was added to anlotinib-treated NCI-H1975 cells or injected into NCI-H1975-derived xenograft models. We found that exogenous CCL2 dramatically offset anlotinib-induced cytotoxicity (including cell apoptosis induction, cell invasion inhibition, cell cycle arrest and inhibition of cell migration) in vitro (supplementary figure S6). Moreover, we evaluated the effect of replenished exogenous CCL2 on angiogenesis-related and proliferation-related proteins in NCI-H1975 in vitro, and we found that extrinsic CCL2 markedly recovered the expression levels of MMP9, CD31 and Ki67 (figure 4a and b).

For the in vivo assessments, we found that both the tumour volume and fold change of tumour volume increased significantly in the anlotinib-treated models injected with CCL2 (figure 4c). Moreover, the survival time declined dramatically after CCL2 administration (figure 4d). Consistent with the observations in vitro, intratumoural MMP9 expression was significantly recovered (figure 4e). Importantly, extrinsic CCL2 significantly neutralised anlotinib-induced anti-angiogenesis (figure $4 \mathrm{f}$ and g). These results indicate that high CCL2 levels not only correlate with vascularisation, but also contribute to offsetting the anlotinib-induced angiogenesis block. Collectively, these results demonstrated that anlotinib had an anti-angiogenesis effect via the inhibition of the CCL2-MMP9 axis (figure 4h) and suggested that anlotinib-induced CCL2 blockade could be associated with the response to anlotinib in refractory advanced NSCLC patients. 


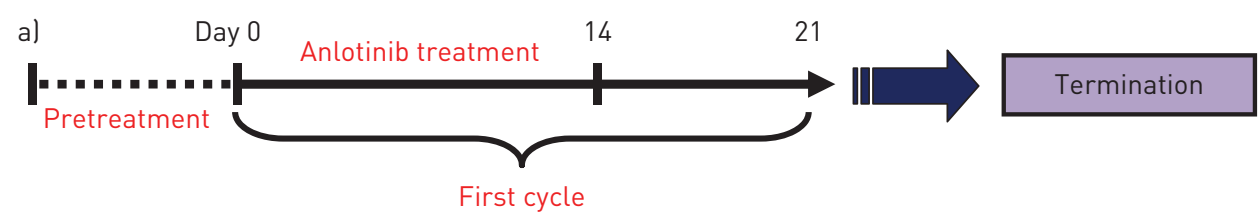

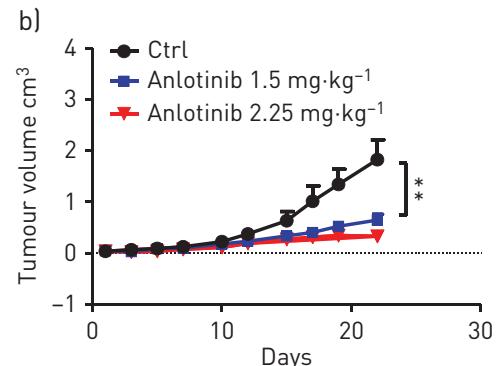

d)

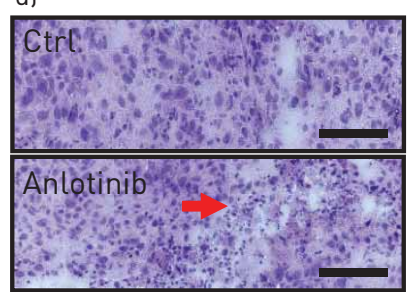

f) GO analysis of downregulated genes Positive regulation of TGF- $\beta 1$ production Wnt signalling pathway, calcium modulating pathway Positive regulation of cell proliferation Positive regulation of toll-like receptor signalling pathway Regulation of VEGF production Positive regulation of protein binding Extracellular matrix organisation Angiogenesis Positive regulation of endothelial cell differentiation Microtubule-based process

g)

\begin{tabular}{|lll|}
\hline $\begin{array}{l}\text { Gene } \\
\text { name }\end{array}$ & $\begin{array}{l}\text { Log }_{2} \text { (fold } \\
\text { change) }\end{array}$ & p-value \\
\hline CCL2 & -4.11297 & $1.59 \times 10^{-2}$ \\
\hline SERPINB4 & -3.20408 & $1.50 \times 10^{-3}$ \\
\hline TUBA4A & -2.87329 & $5.00 \times 10^{-5}$ \\
\hline STRA6 & -2.81923 & $9.00 \times 10^{-4}$ \\
\hline COL7A1 & -2.55164 & $5.00 \times 10^{-5}$ \\
\hline AQP3 & -2.45409 & $6.75 \times 10^{-3}$ \\
\hline CXCL2 & -2.29888 & $1.08 \times 10^{-2}$ \\
\hline MB & -2.2613 & $6.60 \times 10^{-3}$ \\
\hline NBPF16 & -2.24777 & $7.00 \times 10^{-4}$ \\
\hline HIST1H3G & -2.08321 & $1.09 \times 10^{-2}$ \\
\hline
\end{tabular}

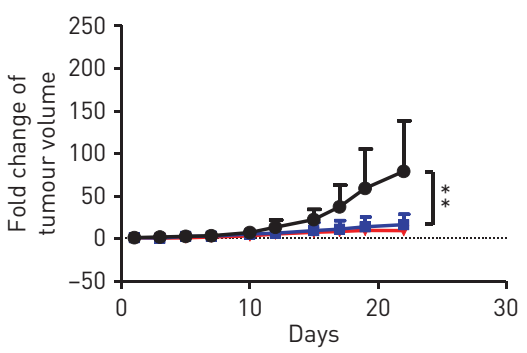

e)

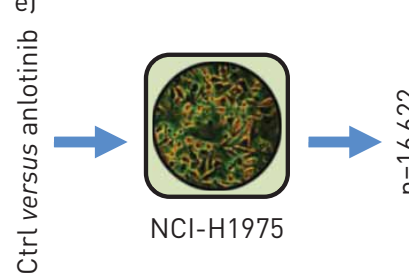

c)

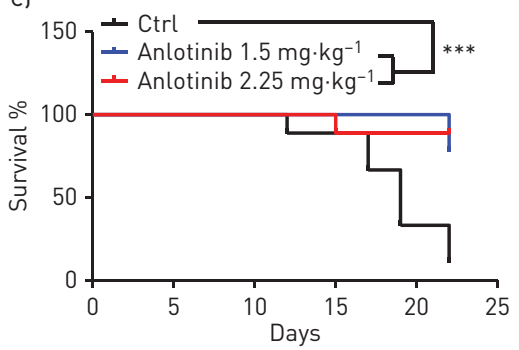

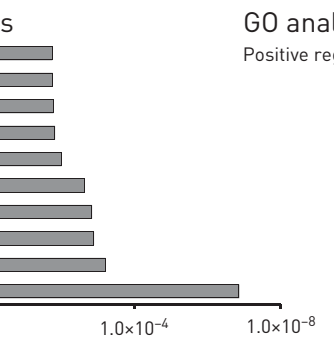

h)

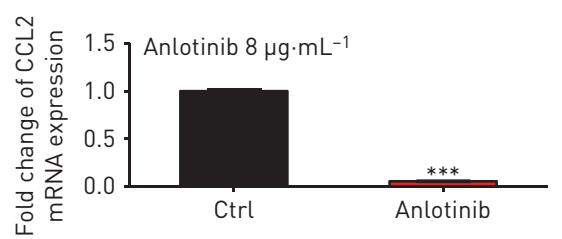

j)

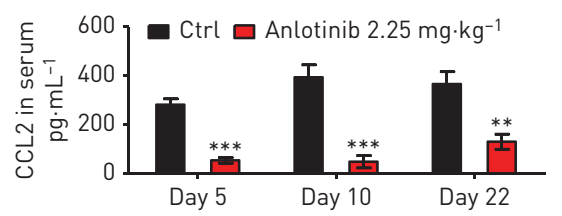

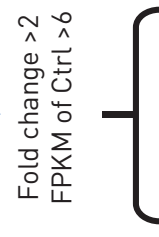

Upregulated genes

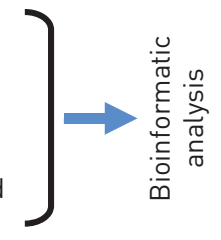



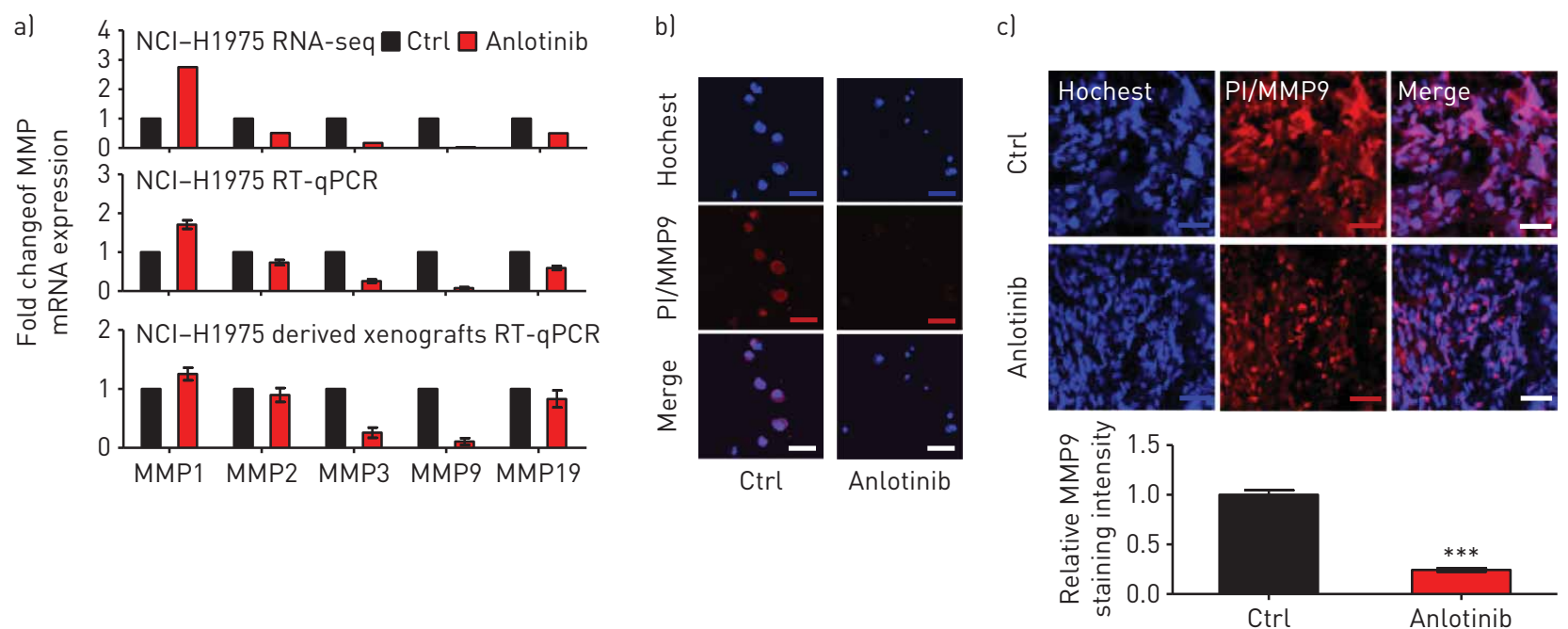
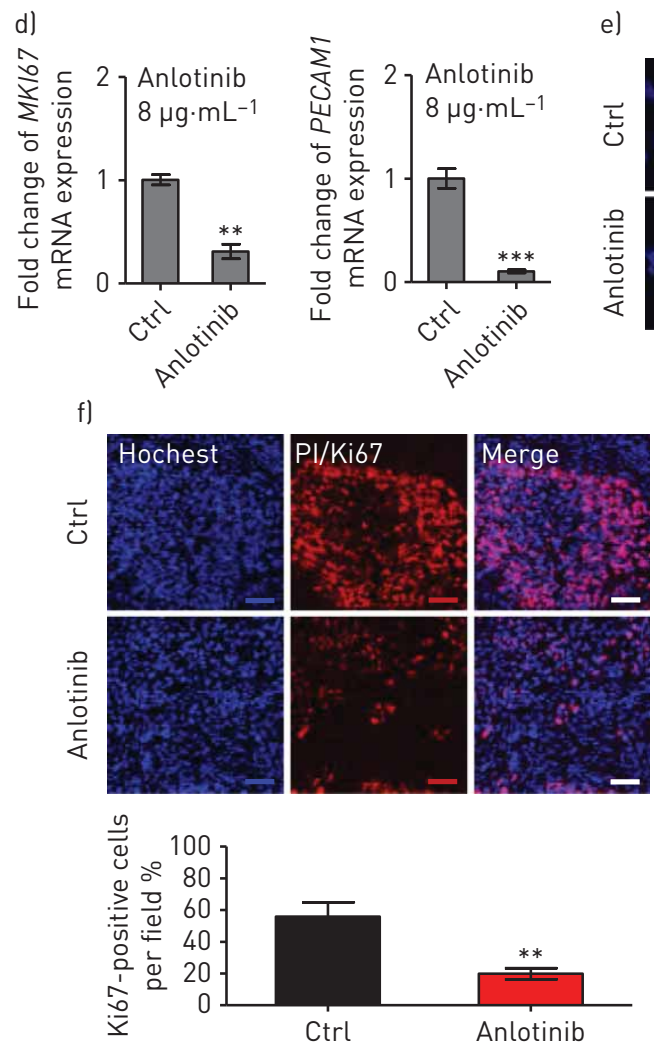

e)

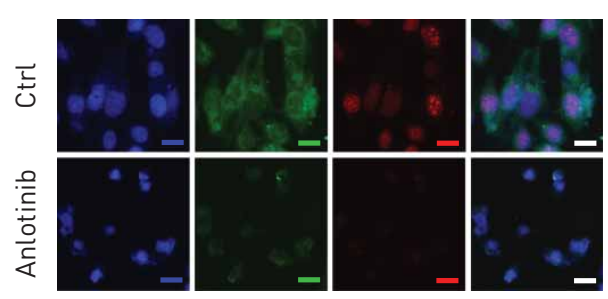

g)
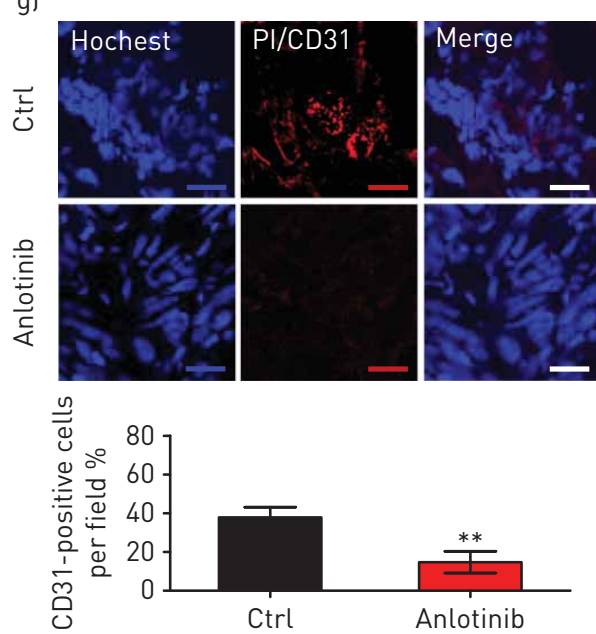

FIGURE 3 Anlotinib inhibits NCl-H1975 cell-derived angiogenesis in vitro and in vivo. a) Matrix metalloproteinase (MMP) mRNA expressions in anlotinib-treated $\left(8 \mu \mathrm{g} \cdot \mathrm{mL}^{-1}\right) \mathrm{NCl}-\mathrm{H} 1975$ cells and anlotinib-treated $\left(2.25 \mathrm{mg} \cdot \mathrm{kg}^{-1}\right) \mathrm{NCl}-\mathrm{H} 1975$-derived xenograft tumours. Data are presented as mean \pm SD, $n=3$. b) Representative images of MMP9-stained NCl-H1975 cells with or without anlotinib (4 $\mu \mathrm{g} \cdot \mathrm{mL}^{-1}$ ) administration. Magnification $10 \times$; scale bars $=100 \mu \mathrm{m}$. Eight images per group. c) Representative images of MMP9-stained NCl-H1975-derived xenograft tumour tissues after anlotinib $\left(2.25 \mathrm{mg} \cdot \mathrm{kg}^{-1}\right)$ treatment for 22 days. Three views of one section per animal. Data are presented as mean \pm SD, $\mathrm{n}=9$ mice per group. Magnification 20x; scale bars $=50 \mu \mathrm{m}$. d) mRNA expression of MKI67 and PECAM1 was evaluated using reverse transcriptase quantitative PCR

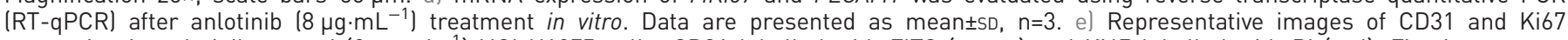
expression in anlotinib-treated $\left(2 \mu \mathrm{g} \cdot \mathrm{mL}^{-1}\right) \mathrm{NCl}-\mathrm{H} 1975$ cells. CD31 labelled with FITC (green) and Ki67 labelled with PI (red). Five images per

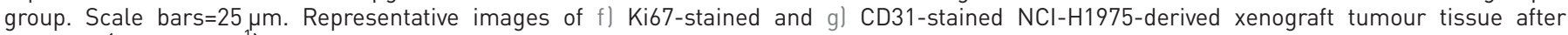
anlotinib $\left(2.25 \mathrm{mg} \cdot \mathrm{kg}^{-1}\right)$ treatment for 22 days. Three views of one section per animal. Data are presented as mean \pm SD, $\mathrm{n}=9$ mice per group. f) Magnification 10x; scale bars $=100 \mu \mathrm{m}$; gl magnification $20 x$; scale bars $=25 \mu \mathrm{m}$. Ctrl: control. ${ }^{* *}$ : $p<0.01$; ${ }^{* * *}$ : $p<0.001$.

Changes in serum CCL2 levels reflect and predict the anlotinib response in refractory advanced NSCLC patients

Given the active involvement of CCL2 in anlotinib treatment, we next asked whether the CCL2 levels could be used to predict the response to anlotinib. Among 28 NSCLC patients treated with anlotinib, we found that serum CCL2 levels in responsive patients $(n=14)$ decreased significantly at best response and 
a)

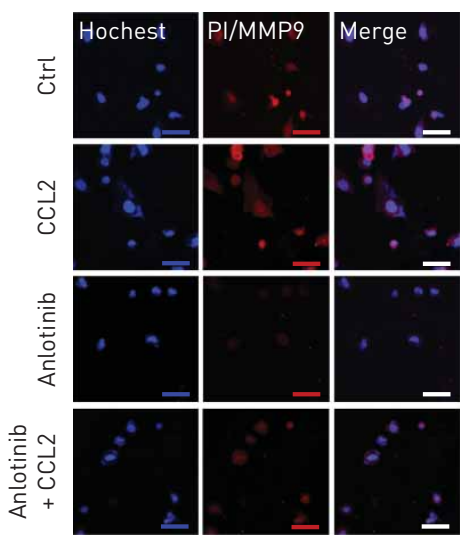

b)

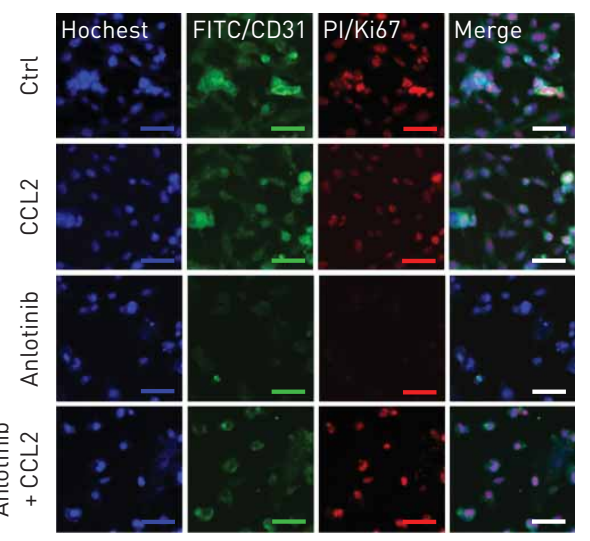

c)
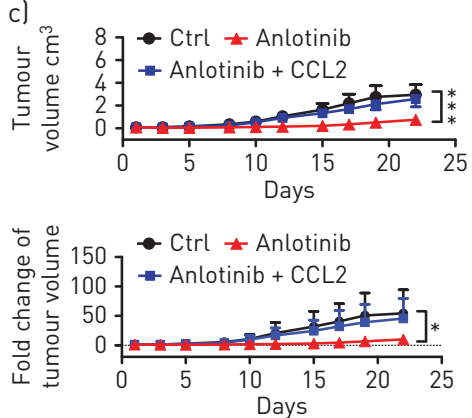

d)

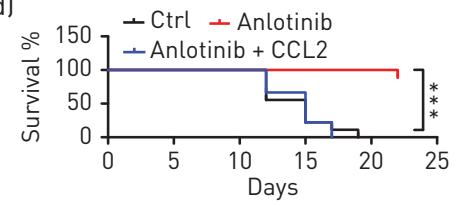

e)

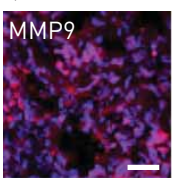

Ctrl

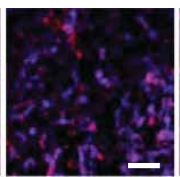

Anlotinib Anlotinib + CCL2

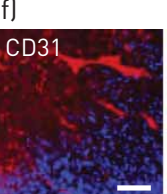

Ctrl

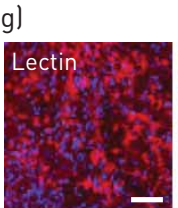

Ctrl

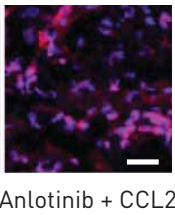

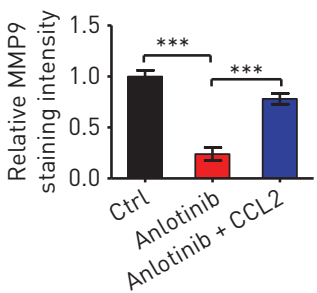

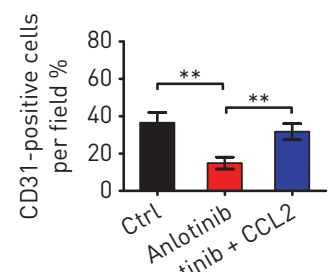
antotim

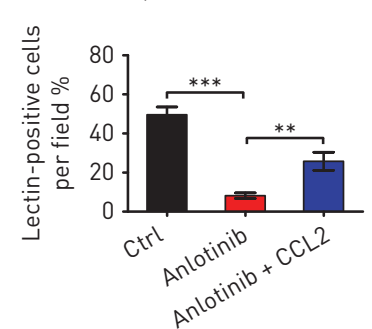

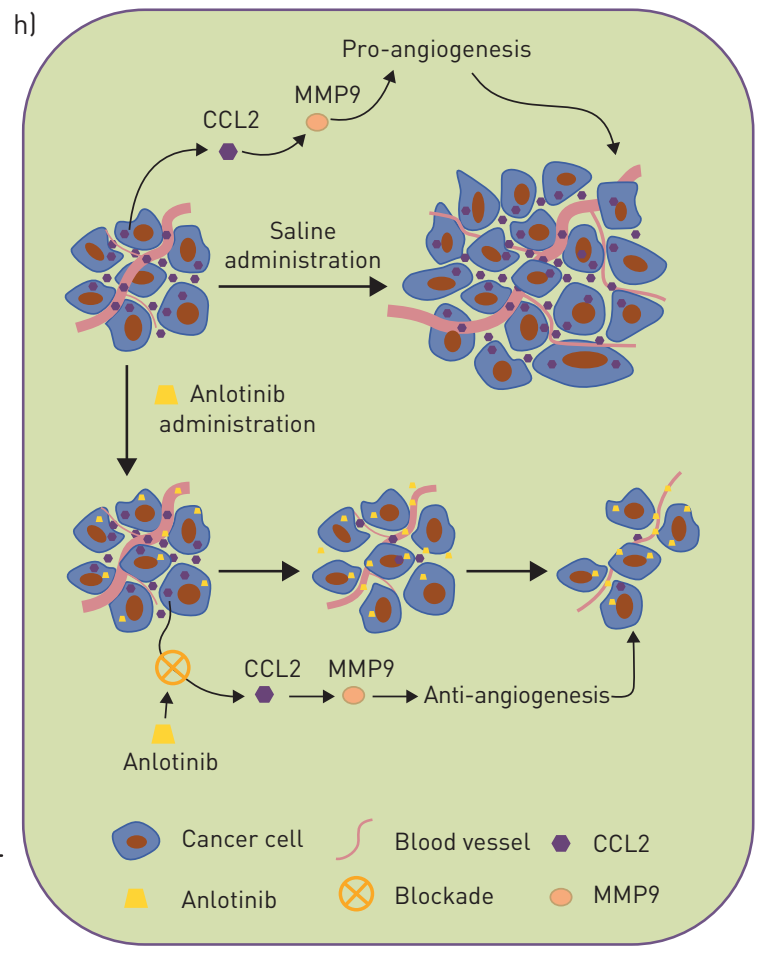

FIGURE 4 Replenishment of exogenous CCL2 recovers anlotinib-induced anti-angiogenesis in vitro and in vivo. $\mathrm{NCl}-\mathrm{H} 1975$ cells were exposed to CCL2 (50 $\mathrm{ng} \cdot \mathrm{mL}^{-1}$ ) and anlotinib $\left(6 \mu \mathrm{g} \cdot \mathrm{mL}^{-1}\right)$, alone or together, for $24 \mathrm{~h}$. a) Representative images of matrix metalloproteinase (MMP)9 expression. Eight images per group. Scale bars $=20 \mu \mathrm{m}$. b) Representative images of CD31 and Ki67 expression. CD31 labelled with fluorescein isothiocyanate (FITC; green) and Ki67 labelled with propidium iodide (PI; red). Six images per group. Scale bars $=100 \mu$ m. c) Measurement of subcutaneous tumour volumes of control (Ctrl), anlotinib $\left(2.5 \mathrm{mg} \cdot \mathrm{kg}^{-1}\right)$ and anlotinib $\left(2.5 \mathrm{mg} \cdot \mathrm{kg}^{-1}\right)+\mathrm{CCL} 2\left(5 \mu \mathrm{gg} \cdot \mathrm{kg}^{-1}\right.$, i.v., alternate days). Data are presented as mean \pm SD, $\mathrm{n}=9$. d) Kaplan-Meier survival curves of mice treated with Ctrl, anlotinib and anlotinib + CCL2; $\mathrm{n=9}$. Representative images of e) MMP9-stained, f) CD31-stained and g) lectin-stained $\mathrm{NCl}$-H1975-derived xenograft tumour tissues on day 22. Three views of one section per animal. Data are presented as mean $\pm S D, n=9$ mice per group. Magnification 20x; scale bars $=50 \mu \mathrm{m}$. h) Schematic of anlotinib inhibition of angiogenesis via blocking the CCL2-MMP9 axis. Tumour cells secrete CCL2 into the tumour microenvironment. CCL2 activates MMP9 expression and then promotes neovascularisation. Administration of anlotinib blocks the CCL2-MMP9 axis in NCl-H1975 cells and NCl-H1975-derived xenografts, contributing to the inhibition of angiogenesis. ${ }^{*}: p<0.05 ;{ }^{* *}: p<0.01 ;{ }^{* *}: p<0.001$.

increased at progressive disease, with 13 responders showing decreased serum CCL2 levels at best response. In nonresponders $(\mathrm{n}=14)$, serum CCL2 levels increased at progressive disease, with 11 nonresponders showing increased CCL2 levels (figure 5a-c). Moreover, we observed the serum CCL2 levels in responders were markedly high compared with those in nonresponders at baseline (supplementary figure S7a). Kaplan-Meier analysis suggested that serum CCL2 levels at baseline 

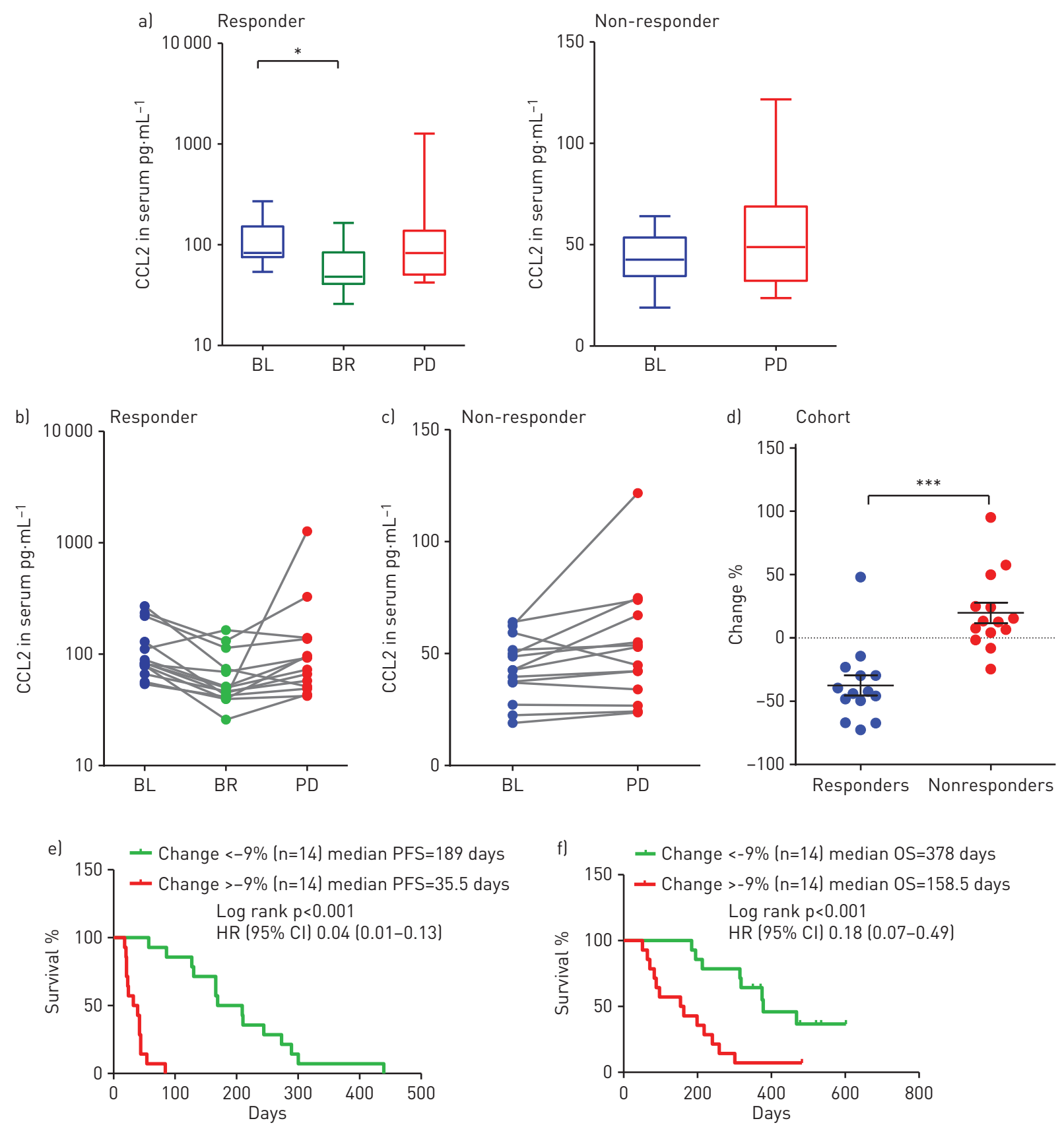

FIGURE 5 Changes in serum CCL2 levels are associated with progression-free survival (PFS) and overall survival (OS) in refractory advanced nonsmall cell lung cancer (NSCLC) patients treated with anlotinib. Advanced NSCLC patients were treated with anlotinib, and serum CCL2 levels were evaluated at baseline (BL), at best response (BR) and at progressive disease (PD). a) The alterative pattern of CCL2 levels in anlotinib-responsive and anlotinib-nonresponsive NSCLC patients. $n=14$. b) Serum CCL2 levels at different time points in anlotinib-responsive NSCLC patients $(n=14)$. c) Serum CCL2 levels were plotted at BL and PD time points for anlotinib-nonresponsive NSCLC patients (n=14). d) Percentages of changes in serum CCL2 in advanced NSCLC patients treated with anlotinib, from baseline to 2 months. Data are presented as mean $\pm S D, n=14$. e) Kaplan-Meier plots of PFS stratified by the changes in CCL2 levels in advanced NSCLC patients treated with anlotinib. $n=28$; cut-off-high: 50\%; cut off-low: 50\%, median (95\% CI) PFS 189 (131-247) days versus 35.5 (25-46) days. f) Kaplan-Meier plots of 0S stratified by the changes in CCL2 levels in advanced NSCLC patients treated with anlotinib. $n=28$; cut-off-high: $50 \%$; cut off-low: $50 \%$, median (95\% CI) 0 S 378 (306-451) days versus $158.5(110-243)$ days. ${ }^{*}: p<0.05 ;{ }^{* * *}: p<0.001$.

potentially predict tumour response in refractory advanced NSCLC patients during treatment with anlotinib (supplementary figure S7b and c).

As mentioned above, in NSCLC cell lines (figure $2 \mathrm{~g}-\mathrm{i}$ and supplementary figure S5), the NCI-H1975-derived xenograft model (figure $2 \mathrm{j}$ and $\mathrm{k}$ ) and NSCLC patients (figure $5 \mathrm{~b}$ ), we observed the remarkable decrease of CCL2 levels upon anotinib treatment. In addition to serum CCL2 levels at baseline, 
we ask whether the change of CCL2 levels could be used to predict anlotinib response. Thus, we examined the changes in serum CCL2 levels after anlotinib therapy. Surprisingly, we found that early changes in CCL2 levels were clearly associated with the response to anlotinib (figure 5d). Subsequently, we used a median cut-off point to assess the anlotinib response. PFS was dramatically longer in NSCLC patients with early decreases in serum CCL2 levels than in those with early increases (figure 5e). Similar results were observed when OS analysis was performed in the cohort using this stratification. Compared with NSCLC patients with increased CCL2 levels after anlotinib therapy, the OS of patients with decreased CCL2 levels was significantly longer (figure $5 f$ ).

\section{Discussion}

As an oral multitargeted TKI, anlotinib exhibits efficacy in various cancers [7, 9-12]. Our clinical evidence suggests that anlotinib prolongs PFS and OS in patients with refractory advanced NSCLC [7, 9, 10]. Although the clinical trial results suggest that anlotinib potentially has an anti-angiogenesis effect to inhibit disease progression, the underlying molecular mechanism is still unclear. In addition, traditional stratification methods for evaluating or predicting responses to new inhibitors are less efficient for identifying the best responders from NSCLC patients. To address these issues, we sought herein to understand the mechanisms of the anlotinib-induced anti-angiogenesis effect and identify a biomarker that could be used to screen anlotinib responders.

Standard therapeutic regimens have been recommended to advanced NSCLC patients as first-line and second-line agents. Third-line and beyond third-line therapy for refractory advanced NSCLC lack a uniform standard, which has been regarded as an intractable problem for the lung cancer research community. Recently, the National Comprehensive Cancer Network has provided third-line therapy regimens, including chemotherapy and immunotherapy, for refractory advanced NSCLC [5]. However, multitargeted TKIs are still worth pursuing. We found that anlotinib could be used as an oral multitarget TKI for third-line or beyond third-line therapy in refractory advanced NSCLC patients $[7,9,10]$. Biomarkers play an important role in drug development for lung cancer therapy. First-generation TKIs provide great benefits to LUAD patients harbouring EGFR mutations (19 deletion or L858R) [23, 24]. Advanced NSCLC patients harbouring 50\% positive PD1/PDL1 expression or a higher tumour mutation burden acquire more benefits from checkpoint inhibitors (such as pembrolizumab, nivolumab and atezolizumab) [25-27]. In this study, we found that LUAD patients harbouring driver gene-positive mutations, especially patients harbouring the EGFR $^{\mathrm{T} 790 \mathrm{M}}$ mutation, obtained more benefit from anlotinib therapy based on OS analysis rather than PFS analysis. The underlying cause of this phenomenon may be attributed to the administration of third-generation TKIs (such as AZD9291) or recovering sensitivity to first-generation TKIs [28, 29]. Although intense efforts have been exerted to identify biomarkers based on known molecular characteristics, it is still difficult to distinguish anlotinib responders from all advanced NSCLC patients.

Understanding the molecular mechanisms of anlotinib could facilitate biomarker identification. Previous studies have indicated that anlotinib selectively inhibits growth factor receptor, including VEGFR (1, 2 and $3)$, PDGFR $(\alpha$ and $\beta$ ) and FGFR (1,2,3 and 4), among others [8-10, 13, 14]. Anlotinib exerts its antitumour effects in tumour cell line-derived xenograft animal models [8]. Furthermore, anlotinib suppresses tumour cell growth through the inhibition of c-Kit, Ret, Aurora-B, c-FMS and DDR1 [15-17]. Elevated CCL2 levels in the tumour microenvironment or blood are associated with tumour angiogenesis $[22,30,31]$. CCL2 activation contributes to angiogenesis and metastasis in lung cancer, breast cancer and pancreatic cancer, among others $[20,21,32]$. The majority of tumours secrete CCL2, a key pro-angiogenesis factor, to stimulate tumour vascularisation and provide nutrition for tumour growth and metastasis [33, 34]. Here, we observed that anlotinib inhibited NCI-H1975 cell proliferation via CCL2 blockade in vitro and in vivo. Moreover, anlotinib-induced tumour necrosis was attributed to angiogenesis inhibition by blocking the CCL2-MMP9 axis in NCI-H1975-derived xenografts models.

Valuable biomarkers contribute to the prediction of responders to new drugs [23-27]. Thus, the identification of a proper biomarker for distinguishing responders from nonresponders to anlotinib is urgent. Here, we observed herein that alterations of serum CCL2 levels were associated with the response to anlotinib therapy in advanced NSCLC. Moreover, we reported that changes in serum CCL2 levels could predict anlotinib responders among refractory advanced NSCLC patients, although serum CCL2 levels at baseline could be used to predict anlotinib responders in this study. However, it is reported that plasma CCL2 levels vary in different cohorts of race, age and smoking [35, 36]. Considering anlotinib induces a remarkable change of CCL2 levels in NSCLC cell lines (figure $2 \mathrm{~g}-\mathrm{i}$ and supplementary figure S5), the NCI-H1975-derived xenograft model (figure $2 j$ and $k$ ) and NSCLC patients (figure $5 b$ ), we found that the change of CCL2 levels, rather than CCL2 levels themselves, could be used to more effectively predict anlotinib response. And a large-scale prospective study should be performed to evaluate its potential as a biomarker for anlotinib therapy. 


\section{Conclusions}

The present study not only reveals an important molecular mechanism of anlotinib-induced anti-angiogenesis via inhibition of the CCL2-MMP9 axis, but also offers insights that serum CCL2 levels could serve as a predictive biomarker for distinguishing anlotinib responders from nonresponders in refractory advanced NSCLC patients receiving third-line or beyond third-line therapies.

Acknowledgements: The authors thank the patients for their participation, and the investigators for their support. We thank Xunqiang Wang and Wei Shi (Chia-tai Tianqing Pharmaceutical Co Ltd, Lianyungang, China) for their help and support.

Author contributions: Experiments were conceived and designed by B.H. Han, J. Lu, L.M. Lu, K. Li and X.D. Zhao. Clinical trials were performed by B.H. Han, K. Li, H. Zhong, T.Q. Chu, X.Y. Zhang, R. Li, J.Y. Sun and R. Zhong. Preclinical experiments were performed by J. Lu, H. Zhong, Y.Q. Yang, M.S. Alam, J. Wu and X.W. Li. Clinical analysis, bioinformatics analysis and statistical analysis were performed by J. Lu, X.Y. Zhang, Y.Q. Lou, J.L. Xu, Y.W. Zhang, X.D. Zhao and J. Wu. The manuscript was written by J. Lu, and revised by X.D. Zhao and B.H. Han.

Conflict of interest: None declared.

Support statement: This work was supported by the Shanghai Leading Talents Program (2013); the Program of System Biomedicine Innovation Center from Shanghai Jiao Tong University (Project No. 15ZH4009); the key Program of Translational Medicine from Shanghai Jiao Tong University School of Medicine (Project No. 15ZH1008); National Natural Science Foundation of China (Project No. 81673015); the Project of the Peak Plateau of Shanghai Jiaotong University School of Medicine - "Research Doctor", (Project No. 20161434); Chia-tai Tianqing Pharmaceutical Co Ltd; and the project of Science and Technology Commission of Shanghai Municipality (Project No. 16140902700). Funding information for this article has been deposited with the Crossref Funder Registry.

\section{References}

1 Zhang Y, Zhang L, Li R, et al. Genetic variations in cancer-related significantly mutated genes and lung cancer susceptibility. Ann Oncol 2017; 28: 1625-1630.

2 Torre LA, Bray F, Siegel RL, et al. Global cancer statistics, 2012. CA Cancer J Clin 2015; 65: 87-108.

3 Grose D, Morrison DS, Devereux G, et al. The impact of comorbidity upon determinants of outcome in patients with lung cancer. Lung Cancer 2015; 87: 186-192.

$4 \quad$ Wistuba II, Gazdar AF. Lung cancer preneoplasia. Ann Rev Pathol 2006; 1: 331-348.

5 Ettinger DS, Wood DE, Aisner DL, et al. Non-small cell lung cancer, version 5.2017, NCCN Clinical Practice Guidelines in Oncology. J Natl Compr Canc Netw 2017; 15: 504-535.

6 Siegel RL, Miller KD, Jemal A. Cancer statistics, 2016. CA Cancer J Clin 2016; 66: 7-30.

7 Han BH, Li K, Wang QM, et al. Efficacy and safety of third-line treatment with anlotinib in patients with refractory advanced non-small-cell lung cancer (ALTER-0303): a randomised, double-blind, placebo-controlled phase 3 study. Lancet Oncol 2017; 18: S3-S3.

8 Sun Y, Niu W, Du F, et al. Safety, pharmacokinetics, and antitumor properties of anlotinib, an oral multi-target tyrosine kinase inhibitor, in patients with advanced refractory solid tumors. J Hematol Oncol 2016; 9: 105.

9 Han $\mathrm{BH}, \mathrm{Li} \mathrm{K}$, Zhao $\mathrm{YZ}$, et al. Anlotinib as a third-line therapy in patients with refractory advanced non-small-cell lung cancer: a multicentre, randomised phase II trial (ALTER0302). Br J Cancer 2018; 118: 654-661.

10 Han BH, Li K, Wang QM, et al. Effect of anlotinib as a third-line or further treatment on overall survival of patients with advanced non-small cell lung cancer: the ALTER 0303 phase 3 randomized clinical trial. JAMA Oncol 2018; 4: 1569-1575.

11 Sun Y, Chi Y, Tan P, et al. Phase II study of anlotinib for treatment of advanced medullary thyroid carcinoma. ASCO Publication 2016: 6015.

12 Chi Y, Sun Y, Cai J, et al. Phase II study of anlotinib for treatment of advanced soft tissues sarcomas. ASCO Publication 2016: 11005.

13 Lin B, Song X, Yang D, et al. Anlotinib inhibits angiogenesis via suppressing the activation of VEGFR2, PDGFR $\beta$ and FGFR1. Gene 2018; 654: 77-86.

14 Xie $\mathrm{C}$, Wan $\mathrm{X}$, Quan $\mathrm{H}$, et al. Preclinical characterization of anlotinib, a highly potent and selective vascular endothelial growth factor receptor-2 inhibitor. Cancer Sci 2018; 109: 1207-1219.

15 Ashton S, Song YH, Nolan J, et al. Aurora kinase inhibitor nanoparticles target tumors with favorable therapeutic index in vivo. Sci Transl Med 2016; 8: 325ra17.

16 Wang $\mathrm{C}$, Chen J, Cao W, et al. Aurora-B and HDAC synergistically regulate survival and proliferation of lymphoma cell via AKT, mTOR and Notch pathways. Eur J Pharmacol 2016; 779: 1-7.

17 Ambrogio C, Gómez-López G, Falcone M, et al. Combined inhibition of DDR1 and Notch signaling is a therapeutic strategy for KRAS-driven lung adenocarcinoma. Nat Med 2016; 22: 270-277.

$18 \mathrm{Lu} \mathrm{J}$, Chen J, Xu N, et al. Activation of AIFM2 enhances apoptosis of human lung cancer cells undergoing toxicological stress. Toxicol Lett 2016; 258: 227-236.

19 Zhang XL, Wu J, Wang J, et al. Integrative epigenomic analysis reveals unique epigenetic signatures involved in unipotency of mouse female germline stem cells. Genome Biol 2016; 17: 162.

20 Bonapace L, Coissieux MM, Wyckoff J, et al. Cessation of CCL2 inhibition accelerates breast cancer metastasis by promoting angiogenesis. Nature 2014; 515: 130-133.

21 An J, Xue Y, Long M, et al. Targeting CCR2 with its antagonist suppresses viability, motility and invasion by downregulating MMP-9 expression in non-small cell lung cancer cells. Oncotarget 2017; 8: 39230-39240.

22 De Palma M, Biziato D, Petrova TV. Microenvironmental regulation of tumour angiogenesis. Nat Rev Cancer 2017; 17: 457-474 
23 Lynch TJ, Bell DW, Sordella R, et al. Activating mutations in the epidermal growth factor receptor underlying responsiveness of non-small-cell lung cancer to gefitinib. N Engl J Med 2004; 350: 2129-2139.

24 Pao W, Miller V, Zakowski M, et al. EGF receptor gene mutations are common in lung cancers from 'never smokers' and are associated with sensitivity of tumors to gefitinib and erlotinib. Proc Natl Acad Sci USA 2004; 101: 13306-13311.

25 Rizvi NA, Hellmann MD, Snyder A, et al. Cancer immunology. Mutational landscape determines sensitivity to PD-1 blockade in non-small cell lung cancer. Science 2015; 348: 124-128.

26 Brahmer J, Reckamp KL, Baas P, et al. Nivolumab versus docetaxel in advanced squamous-cell non-small-cell lung cancer. N Engl J Med 2015; 373: 123-135.

27 Herbst RS, Baas P, Kim DW, et al. Pembrolizumab versus docetaxel for previously treated, PD-L1-positive, advanced non-small-cell lung cancer (KEYNOTE-010): a randomised controlled trial. Lancet 2016; 387: 1540-1550.

28 Parra HJS, Noto L, Galetta D, et al. A phase II, noncomparative, open label, multicentre, study of AZD9291 in patients with locally advanced or metastatic EGFR mutated "T790M undetectable or unknown" non-small cell lung cancer (stage IIB-IV) after no immediate prior EGFR TKI (OSIRIS study). J Clin Oncol 2017; 35: TPS9107.

29 Chang GC, Tseng CH, Hsu KH, et al. Predictive factors for EGFR-tyrosine kinase inhibitor retreatment in patients with EGFR-mutated non-small-cell lung cancer - a multicenter retrospective SEQUENCE study. Lung Cancer 2017; 104: 58-64.

30 Fridlender ZG, Buchlis G, Kapoor V, et al. CCL2 blockade augments cancer immunotherapy. Cancer Res 2010; 70: 109-118.

31 Devaud C, John LB, Westwood JA, et al. Immune modulation of the tumor microenvironment for enhancing cancer immunotherapy. Oncoimmunology 2013; 2: e25961.

32 Sanford DE, Belt BA, Panni RZ, et al. Inflammatory monocyte mobilization decreases patient survival in pancreatic cancer: a role for targeting the CCL2/CCR2 axis. Clin Cancer Res 2013; 19: 3404-3415.

33 Tas SW, Maracle CX, Balogh E, et al. Targeting of proangiogenic signalling pathways in chronic inflammation. Nat Rev Rheumatol 2016; 12: 111-122.

34 Kudo-Saito C, Shirako H, Ohike M, et al. CCL2 is critical for immunosuppression to promote cancer metastasis. Clin Exp Metastasis 2013; 30: 393-405.

35 McDermott DH, Yang Q, Kathiresan S, et al. CCL2 polymorphisms are associated with serum monocyte chemoattractant protein-1 levels and myocardial infarction in the Framingham Heart Study. Circulation 2005; 112: 1113-1120.

36 Bielinski SJ, Pankow JS, Miller MB, et al. Circulating MCP-1 levels shows linkage to chemokine receptor gene cluster on chromosome 3: the NHLBI Family Heart Study follow-up examination. Genes Immun 2007; 8: 684-690. 